\title{
Flow characteristics of gas-liquid-particle mixing in a gas-stirred ladle system with throughflow
}

\author{
S. Torii, W.-J. Yang
}

\begin{abstract}
An experimental study is performed on air-liquid-particle mixing, resulting from an air-particle mixture injected into a liquid flowing through a slender ladle. Flow visualization combined with image processing is employed to investigate the bubble and particle behavior at the nozzle outlet. Effort is directed to particle discrimination in both the liquid and the bubbles to determine particle distribution, which affects the mixing performance of gas bubbles, solid particles and liquid. A real-time movement of bubble and particle behavior can be visualized by means of image processing with the use of a slow-motion video recording. It is disclosed that the particles injected through the nozzle may stick on the inner surface of the gas bubble, break through the bubble surface, or mingle with the gas stream to form a two-phase jet, depending on the particle-to-gas mass flow rate ratio. It is observed that when a solid-gas two-phase jet penetrates deeper in the horizontal direction, the particles and bubbles rise along the vertical sidewall and simultaneously spread in the transverse direction, thus promoting a better liquid-particle mixing. The application of the slow-motion video recording results in quantitative evaluations of both the penetration depth of particles or of gas-particles from the injection nozzle and the velocity distribution along the sidewall.
\end{abstract}

\section{List of symbols}

$B \quad$ Width of water vessel, $\mathrm{m}$

$B_{n} \quad$ Nozzle location on bottom surface of water vessel, $\mathrm{m}$

$d_{o} \quad$ Diameter of a gas-particle injection nozzle, $\mathrm{m}$

$H \quad$ Height of water vessel, $\mathrm{m}$

$H_{n} \quad$ Nozzle location on vertical surface of water vessel, $\mathrm{m}$

$L \quad$ Penetration length of particles or of particles and gas from the nozzle, $m$

Received: 10 October 1994/Accepted: 30 March 1995

S. Torii ${ }^{1}$, W.-J. Yang

Department of Mechanical Engineering and Applied Mechanics, University of Michigan, Ann Arbor, Michigan, 48109, USA

\footnotetext{
${ }^{1}$ Visiting scholar on leave from the Mechanical Engineering Department, Kagoshima University, Kagoshima, Japan
}

The work reported was supported by the National Science Foundation under the Grant No. CTS-8921584

\author{
$Q_{g} \quad$ Volumetric flow rate of gas, $\mathrm{m}^{3} / \mathrm{s}$ \\ $Q_{\text {I }} \quad$ Volumetric flow rate of water, $\mathrm{m}^{3} / \mathrm{s}$ \\ $Q_{S} \quad$ Volumetric flow rate of particle, $\mathrm{m}^{3} / \mathrm{s}$ \\ $R e_{g} \quad$ Gas Reynolds number based on inner diameter of the \\ air-particle injection nozzle \\ $t$ Time, sec. \\ $W$ Thickness of water vessel, $\mathrm{m}$ \\ $x \quad$ Transverse coordinate, $\mathrm{m}$ \\ $y \quad$ Longitudinal coordinate, $\mathrm{m}$ \\ $\Gamma \quad$ Mass flow rate ratio of particles to gas
}

1

\section{Introduction}

The injection of powders into molten metals, which is called injection metallurgy, is of great importance in metallurgical refining operations. Typical applications include the desulphurization of iron or steel, the modification of inclusion morphology and composition, and the decarburization of steel by argon in combination with blowing practices (Szekely et al. 1976; Grevet et al. 1982; Sahai and Guthrie 1982a, b; Mazumdar and Guthrie 1985). In such industrial injection processes, powders are pneumatically transported, and then injected into a molten metal through a submerged lance. The carrier gas, while rising as a plume to the free surface, induces circulatory flows in the fluid within the vessel, thereby helping to promote metal homogeneity.

At present, ladle metallurgical operations with throughflow are still in the developmental stage. Only a proto-type device is known to have been tested for possible application in the continuous casting of steel (Torii and Yang 1993). Molten metal mixed with particles of chemical agent flows steadily through the ladle into which a stream of inert gas is injected for stirring purpose. The slugs of the chemical agent rise after a reaction with the impurity in the melt and exit through a port at the ladle top. Torii and Yang (1992) proposed a physical model to simulate the operation of the pilot plant, in which the ladle was made slender in geometry to produce a twodimensional flow. Because its shape is convenient to the experimental investigation and numerical simulation of the mixing performance in the system. The solid particles were introduced into the ladle through injection with gas, instead of being carried by the throughflow. The mixing performance was investigated using the direct photographic method. The following results were obtained:

(i) Mixing characteristics are mainly affected by the gas nozzle location and the flow rates of gas and particle and its efficient performance is achieved if the gas at a higher flow rate 
is injected with particles through an air nozzle near the bottom corner of the ladle wall on the liquid inlet side.

(ii) In two-dimensional ladles with the same width, the mixing performance in a rectangular container (aspect ratio of 2 ) is superior to that of a square one (aspect ratio of unity).

(iii) The mixing performance is better in the vertical than horizontal injections because the buoyant force acting on the bubble is aided by the injection force in the vertical case, forcing the bubble surface to ascend faster.

(iv) The effect of throughflow rates on the gas-particle mixing is rather minor.

The method was extended to investigate the bubble and particle behavior and mixing performance at the outlet of an air injection nozzle (Torii and Yang 1991). It was disclosed that:

(i) The bubble shape as well as the mixing performance depend on both the particle flow rate and the nozzle location.

(ii) A larger deformation in the bubble shape occurs at a higher particle flow rate, when the injection nozzle is horizontally positioned at the lower end of the sidewall.

(iii) In addition to the effects of the buoyancy and viscous forces, the distortion of bubble shape can also be attributed to contamination of bubble surface in the presence of particles which results in a reduction of surface tension.

(iv) The inertial force of traveling particles causes the bubble to burst. The information provides an important clue in understanding the mechanisms of the air-particle mixing in a ladle and a guide in developing a numerical model for the submerged injection of a gas-particle mixture. Moreover, it also is important to confirm the above experimental results by means of the other methods.

To further develop the system proposed above, it needs to explore a microscopic view of mixing characteristics within it. This requires understandings of the penetration-dispersion behavior of solid particles into the molten metal as well as the trajectory and penetration depth of the solid-gas, two-phase jet. Due to the complexity of such a flow, it is very difficult to obtain quantitative and/or accurate results. As the first step, attention is paid to the behavior of gas bubble and particles when a gas-particle mixture is introduced into the ladle.

The present study employs the direct photographic method and the image processing procedure to investigate the penetration-dispersion behavior of particle-carrying gas into the molten metal. Emphasis is placed on the discrimination of particles in both the bubble and the liquid.

\section{2}

\section{Experimental apparatus}

The experimental apparatus employed in the present study is depicted in Fig. 1. It consisted of a slender rectangular tank (simulating a ladle with a 2-D flow field in the $x$ and $y$ directions) with two large side walls which was made of acrylic windows for illumination and observation. A ladle with the dimensions of $W=0.05 \mathrm{~m}, B=0.2 \mathrm{~m}$ and $H=0.4 \mathrm{~m}$ was used as the processing tank, in which fluid flow was treated as two-dimensional. This situation was verified by using the thin light sheet of a He-Ne laser $(5 \mathrm{~mW}$ ) beam. It cut vertically through the midpoint of the slender sidewall and then the rising bubble and its bursting were uniformly observed on the sheet, achieving in a 2 -D flow. Four light sources $(4 \times 200 \mathrm{~W}$

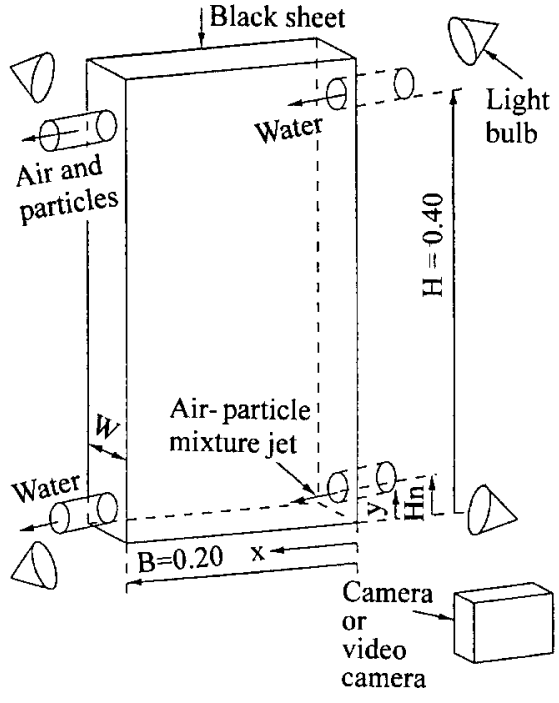

Fig. 1. A schematic of flow visualization apparatus

bulbs) were used for illumination. In order to prevent light reflection from the acrylic windows, the light bulbs were placed on both sides of the tank, as shown in the figure, with a black sheet pasted on the rear sidewall. Water was used to simulate the molten metal, because the kinematic viscosity of water as the most important property is essentially equivalent to that of molten steel at $1873 \mathrm{~K}$ (Sahai and Guthrie 1986). It flowed steadily at a volumetric rate of $Q_{l}$ into and out of the tank via the inlet and exit pipes of $0.01 \mathrm{~m}$ inner diameter. A stream of air (simulating the carrier gas) $Q_{g}$, mixed with neutrally solid particles (simulating desulphurization or deoxidizing agents) $Q_{S}$, was injected into the tank via a submerged nozzle with a $0.005-\mathrm{m}$ inner diameter. The air nozzle was located at the midpoint of the slender side, horizontally installed at the lower end of the right sidewall, $H_{n} / H=0.05$. For the purpose of evaluating the local velocity of water flow in the ladle, the solid particles were used as water seeds. Polypropylene spheres of $100 \mu \mathrm{m}$ in diameter, whose size is smaller than that used in the previous study (Torii and Yang 1992), were employed. Their density $\left(910 \mathrm{~kg} / \mathrm{m}^{3}\right.$ at $\left.297 \mathrm{~K}\right)$ is comparable to that of water and particles used here yield a slight drag, so that the stream of particles may be approximately recognized as gas-particleliquid mixing flow.

In the present study, the image processing technique was utilized to observe the bubble morphology, mixing performance and mechanics of mixing of the three phases (gas-particle-liquid) near the injection nozzle. Volumetric flow rate of water was set at $Q_{l}=3.31 \times 10^{-5} \mathrm{~m}^{3} / \mathrm{s}$ throughout the experiments, because the effect of throughflow rate on mixing is minor, as mentioned in the introduction. The ranges of the parameters for the present study are volumetric flow rates of gas $Q_{g}=0.90 \times 10^{-4}, 2.10 \times 10^{-4}, 2.45 \times 10^{-4}, 2.80 \times 10^{-4}$, $3.15 \times 10^{-4}$, and $3.51 \times 10^{-4} \mathrm{~m}^{3} / \mathrm{s}$; and volumetric flow rates of particles, $Q_{S}=0.0-12.50 \times 10^{-6} \mathrm{~m}^{3} / \mathrm{s}$. The corresponding maximum gas Reynolds number, $R e_{g}$ is 5,730 . Higher $R e_{g}$ could not be obtained due to the limitation of the water vessel size employed here. Experiments were performed under the condition of $T=293 \mathrm{~K}$ and $1 \mathrm{~atm}$. Thus $v=0.156 \times 10^{-4} \mathrm{~m}^{2} / \mathrm{s}$ and $\rho=1.166 \mathrm{~kg} / \mathrm{m}^{3}$ are used as thermal properties of air. 


\section{Digital image processing}

Direct photography was used in flow visualization. The particle-carrying gas jet in the water tank was photographed by a $35 \mathrm{~mm}$ single-lens reflex A-1 Canon camera with $50 \mathrm{~mm}$ Nikon lenses. Kodak Tri-X black and white films were exposed at $1 / 30 \mathrm{sec}$. The change in the bubble and jet morphology was directly recorded by a video camera with $640 \times 480$ resolution, which can record a fast-moving object at $1 / 4000 \mathrm{sec}$. shutter speed and equips a device capable of reading up to $1 / 60 \mathrm{sec}$. These image data were supplied to an automatic data collection system, as illustrated in Fig. 2. The system consisted of a video camera, a time device, a video cassette recorder, a Vicom image digitizer (a digitized image has an active area of 512 pixels horizontally by 480 pixels vertically), a display with $512 \times 480$ pixels, a 32-bit IBM personal computer, and a laser printer (printing dots at $300 \mathrm{DPI})$. Generally speaking, the image processing becomes more difficult if the movement of the small particles is recorded. In the present study, since a video camera records the restricted region near the gas injection nozzle, the image processing technique can be employed.

4

\section{Results and discussion}

\section{1}

\section{Bubble and particle behaviour at the outlet of an air injection nozzle}

Figures $3 \mathrm{a}, \mathrm{b}$ and $\mathrm{c}$ exhibit particle behavior following injection at a gas flow rate $Q_{g}$ of $0.90 \times 10^{-4} \mathrm{~m}^{3} / \mathrm{s}$ with particle flow rates $Q_{S}$ of $0,1.50 \times 10^{-6}$ and $10.00 \times 10^{-6} \mathrm{~m}^{3} / \mathrm{s}$, respectively. Each results from an image processing. Particles and their clusters appear in white. At a low particle flow rate, Fig. 3b, particles stick to the inner surface of the bubble whose morphology resembles that in the absence of particle injection, Fig. 3a. At a higher particle flow rate, Fig. $3 c$, however, the bubble deforms significantly, resulting from particles penetrating the bubble surface and dispersing into the surrounding liquid. Figures 4a, $\mathrm{b}$ and $\mathrm{c}$ show the bubble morphology at a gas flow rate of $3.51 \times 10^{-4} \mathrm{~m}^{3} / \mathrm{s}$ with the particle flow rates of $0,4.00 \times 10^{-6}$ and $10.00 \times 10^{-6} \mathrm{~m}^{3} / \mathrm{s}$, respectively. There is no particle penetrating the bubble surface as seen previously in Fig. 3c. In contrast, a particle-laden gas is observed in both Figs. $4 \mathrm{~b}$ and $c$. This is because both jets are formed by a horizontal migration of a particle-carrying gas stream at a high flow rate which possesses a large inertia force. As the particle flow rate increases, the solid-gas, two-phase jet penetrates deeper into the bulk liquid. It is concluded from the above results that the penetration depth of particles or mixed-phase jet and the bubble morphology are affected by the flow rates of gas and particles. Both Figs. 3 and 4 are images obtained at an arbitrary instant. Since these particle-carrying bubble phenomena are too chaotic to be visualized by the naked eye, it is necessary to record a continuous motion of both phases in order to comprehend the behavior of gas and particles in the liquid. To achieve this objective, a real-time image processing is undertaken here by utilizing the slow-motion function of a video deck.

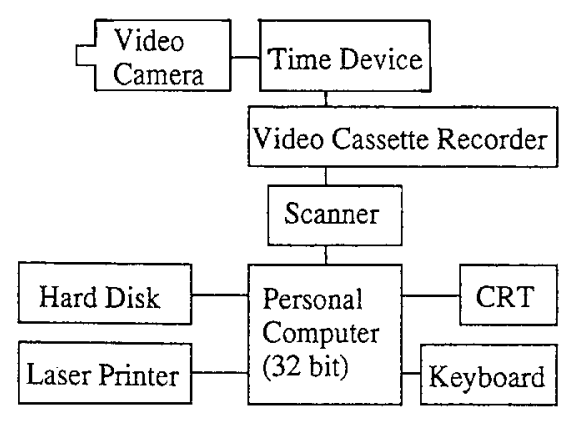

Fig. 2. A real-time image processing system
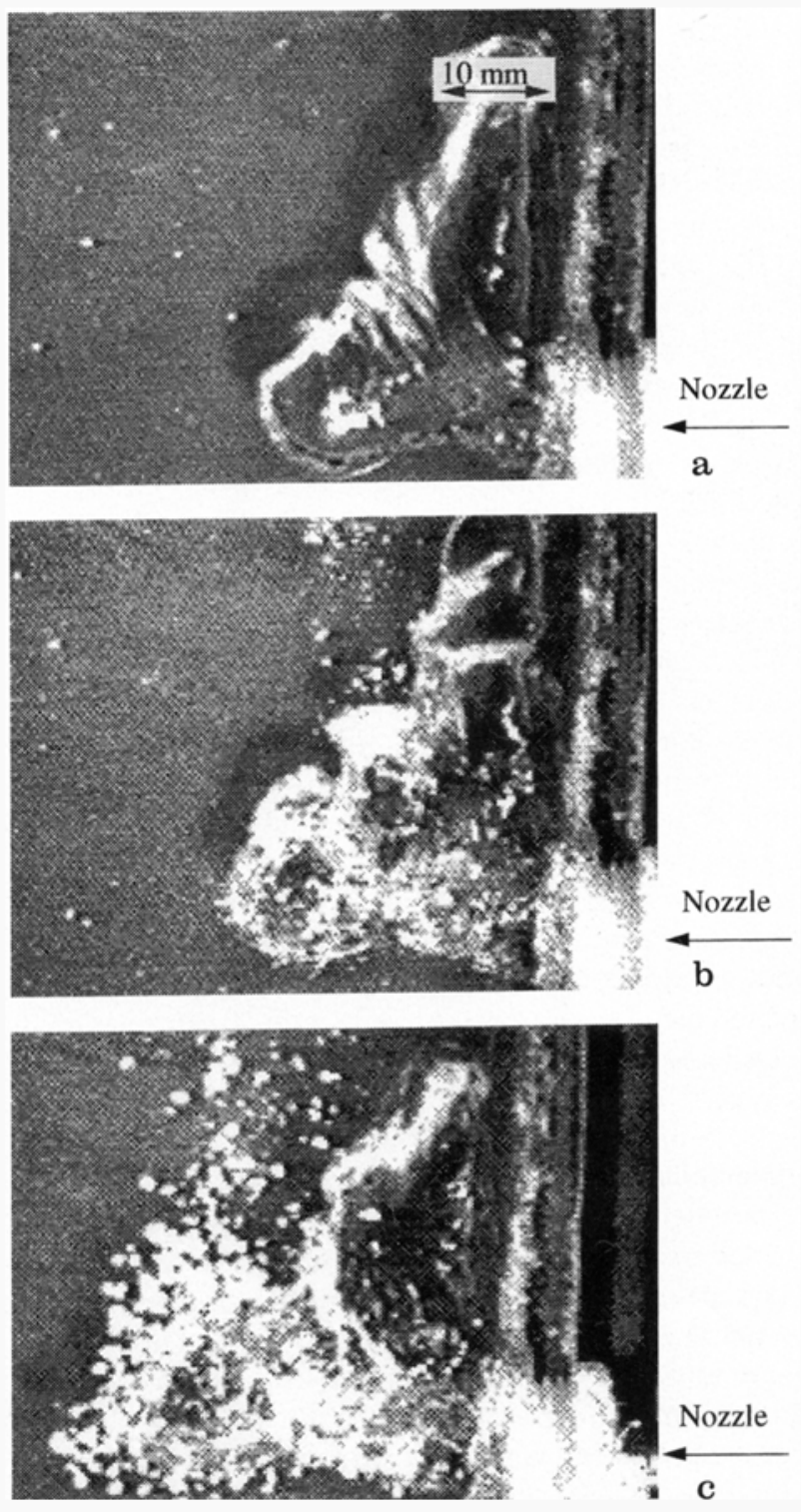

Fig. 3a-c. Image processing of particle distribution in an air bubble for $Q_{g}=0.90 \times 10^{-4} \mathrm{~m}^{3} / \mathrm{s}$ with a horizontal air nozzle at the lower end of right sidewall, $H_{n} / H=0.05$ 


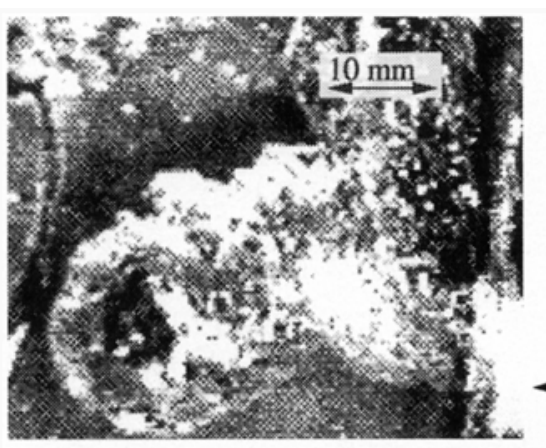

Nozzle

a
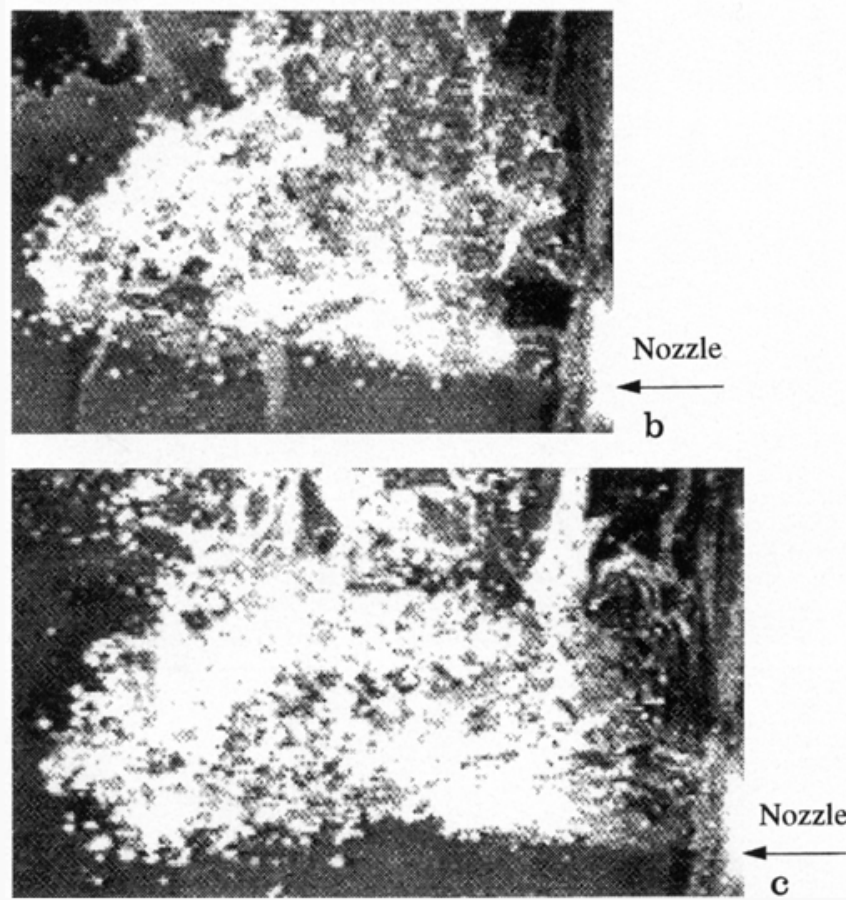

Fig. 4a-c. Image processing of particle distribution in an air bubble for $Q_{g}=3.51 \times 10^{-4} \mathrm{~m}^{3} / \mathrm{s}$ with a horizontal air nozzle at the lower ans of right sidewall, $H_{n} / H=0.05$

Figure 5 illustrates both the horizontal and vertical motion of gas and particles under the same experimental condition as Fig. 4c. Each frame is taken at an interval of $0.017 \mathrm{sec}$. After a particle-laden gas is horizontally injected through a nozzle into the liquid at $t=0 \mathrm{sec}$, the bubble grows and ascends along the sidewall. Simultaneously, a portion of the bubble moves horizontally together with particles due to the action of inertia force, thus forming a solid-gas, two-phase jet as seen in Fig. 5b. Although the jet further penetrates in the horizontal direction, its motion ceases after $t=0.034 \mathrm{sec}$. Therefore, the particles and the surrounding gas begin to ascend due to the buoyancy force, with the jet bending upward, as shown in Fig. 5h. At $t=0.153$, i.e. Fig. 5 j, the jet morphology is disrupted by the succeeding jet which penetrates in the horizontal direction. It is concluded from the above results that a particle-carrying jet does not maintain its shape which varies with time.

An attempt, based on the image data, is made to evaluate the horizontal penetration distance of particles or of gas and particles from the nozzle. From an instantaneous photograph of a bending jet, as seen in Fig. 6, Engh (1979) defined the jet penetration depth as the length from the nozzle exit to the position where its inclination from the horizontal directions becomes $1 / 2$. However, this definition is not appropriate since the jet morphology varies with time. This paper defines it as the distance from the nozzle exit to the location where a particle or particle-gas, mixed phase jet loses its horizontal momentum. This distance is repeatedly measured with time and its arithmetic average value is taken as the penetration depth, $L$. Figure 7 plots the penetration depth against the gas Reynolds number, $R e_{g}$, based on the nozzle diameter, $d_{o}$, as the characteristic length. The particle-to-gas mass flow rate ratio, $\Gamma$, is the parameter in the plot. The correlation equation for the mean penetration depth is found to be

$L=0.000175(1+0.691 \Gamma) \operatorname{Re}_{g} d_{o}$.

Equation (1) is superimposed on the figure as solid lines. It is observed that with $\Gamma$ fixed, $L$ is directly proportional to $R e_{g}$ and that the slope of the straight lines increases with an increase in $\Gamma$. Although the effects of air nozzle diameter and density and diameter of particles on the penetration depth may be considered, they are investigated by using a simple multiphase fluid dynamic model (Torii and Yang 1995). It was disclosed from this numerical result that (i) the penetration depth is not substantially affected by the air nozzle size, (ii) the penetration depth is increased with an increase in the gas flow rate and this trend is induced by $\Gamma$, and (iii) an augmentation of penetration of the mixing jet into a liquid becomes larger as the particle density is equal to that of a liquid.

\section{2}

Rising bubble and particle behavior along the vertical sidewall After particles are pneumatically injected through the horizontal nozzle as seen in Fig. 5, the behavior of the bubbles and particles along the vertical right sidewall in the tank is shown in Fig. 8. The operating conditions, the air flow rate and their injection location, are the same as those of Fig. 4.

Figures $8 \mathrm{a}, \mathrm{b}$ and $\mathrm{c}$, with a horizontal injection nozzle at the lower end of the sidewall $H_{n} / H=0.05$, correspond to the particle flow rates of $Q_{S}=0,4.00 \times 10^{-6}$, and $10.00 \times 10^{-6} \mathrm{~m}^{3} / \mathrm{s}$, respectively. Particles and their clusters appear white. As the particle flow rate was increased, the solid-gas mixing jet penetrates deeper into the liquid, resulting in a homogeneous particle distribution along the sidewall. So far, results present only the still images of the behavior of particles and bubbles at an arbitrary time. A slow-motion function of the video cassette recorder was employed to observe the phenomena of rising bubbles and particles.

Figure 9 depicts the bubble and particle movement under the same operating condition as in Fig. $8 \mathrm{c}$. Figures $9 \mathrm{a}, \mathrm{b}$ and $c$ correspond to their motion at $t=0,0.034$ and $0.068 \mathrm{sec}$, respectively. Particles in the figures are shown as pure white spots. After the particle-air mixture was injected through a horizontal nozzle at $t=0 \mathrm{sec}$, the particle-laden gas bubbles became larger and at the same time the jet formed by it traveled in the horizontal direction due to inertial force, as shown in the lower part of Fig. 9b. After that, the particles rose along the sidewall and simultaneously spread in the transverse direction. This situation becomes clearer if attention is focused on the 

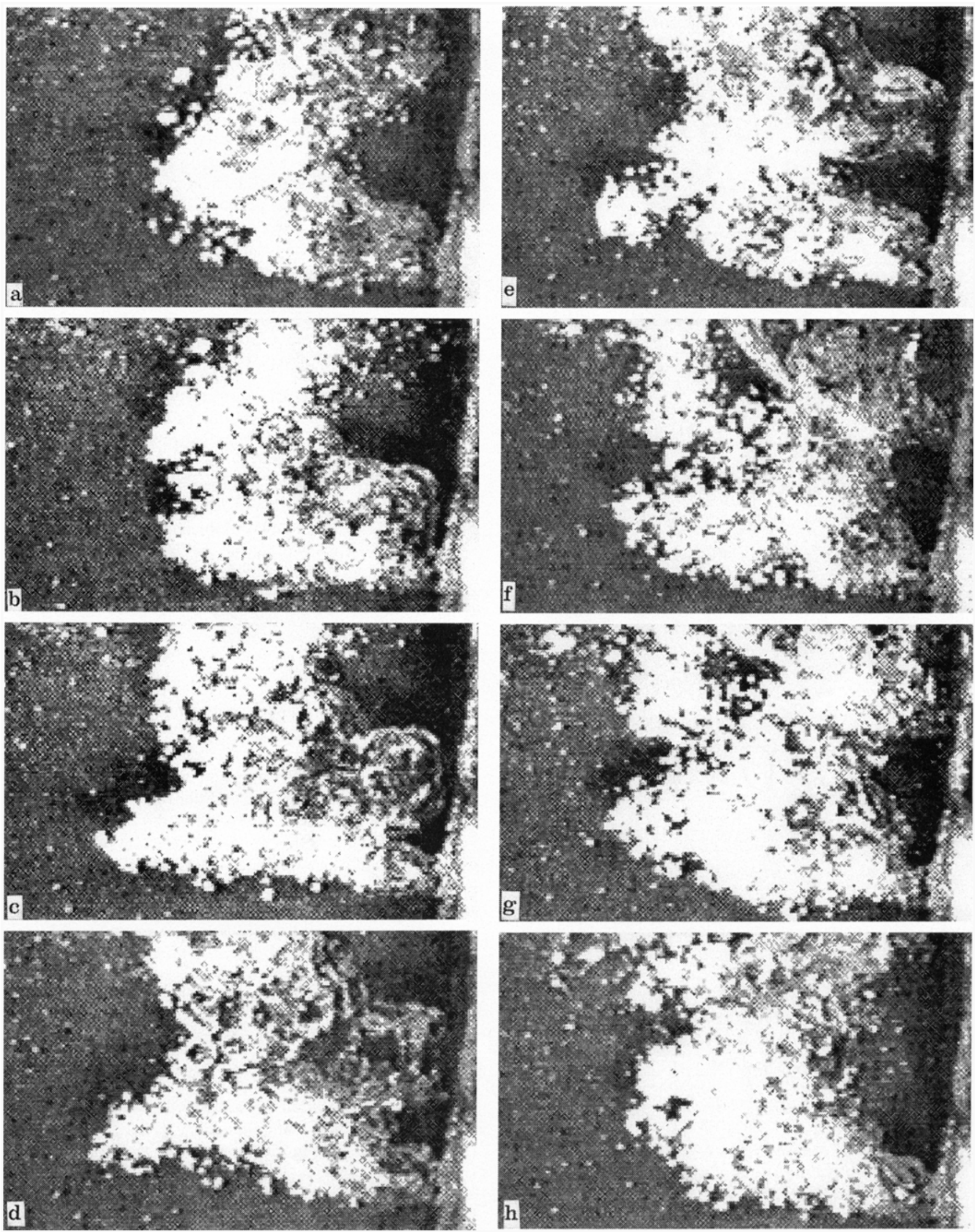

Fig. 5a-l. A real-time image processing of bubble and particle behavior for $Q_{g}=3.51 \times 10^{-4} \mathrm{~m}^{3} / \mathrm{s}$ and $Q_{S}=10.00 \times 10^{-6} \mathrm{~m}^{3} / \mathrm{s}$ with a horizontal air nozzle at $H_{n} / H=0.05$ 

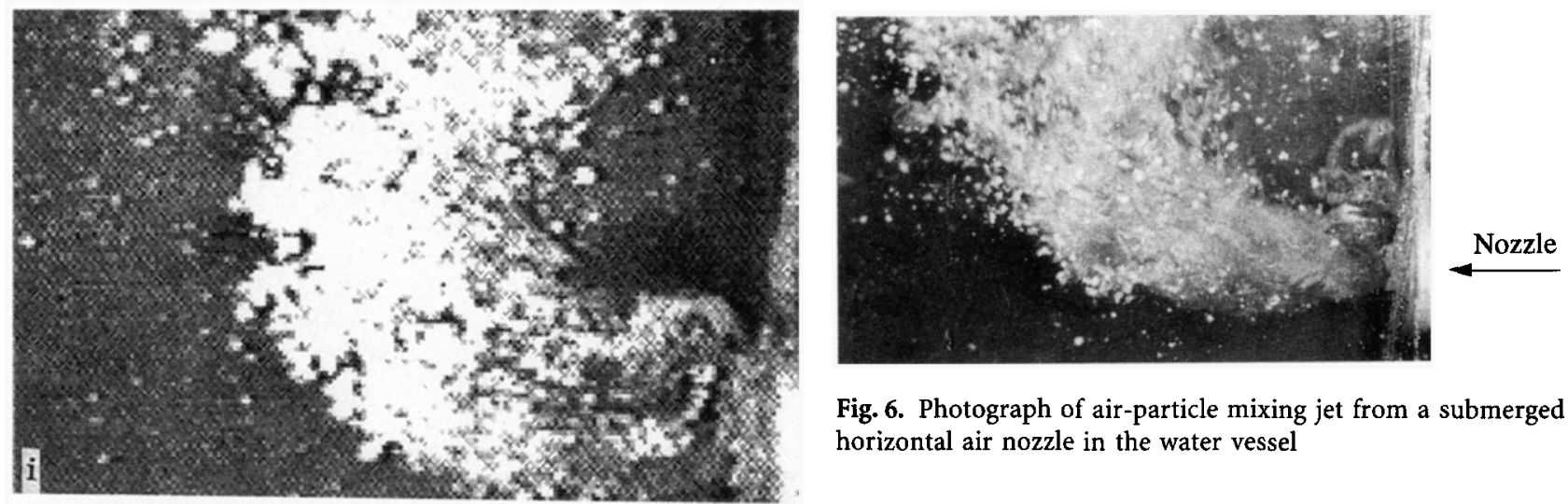

Fig. 6. Photograph of air-particle mixing jet from a submerged horizontal air nozzle in the water vessel
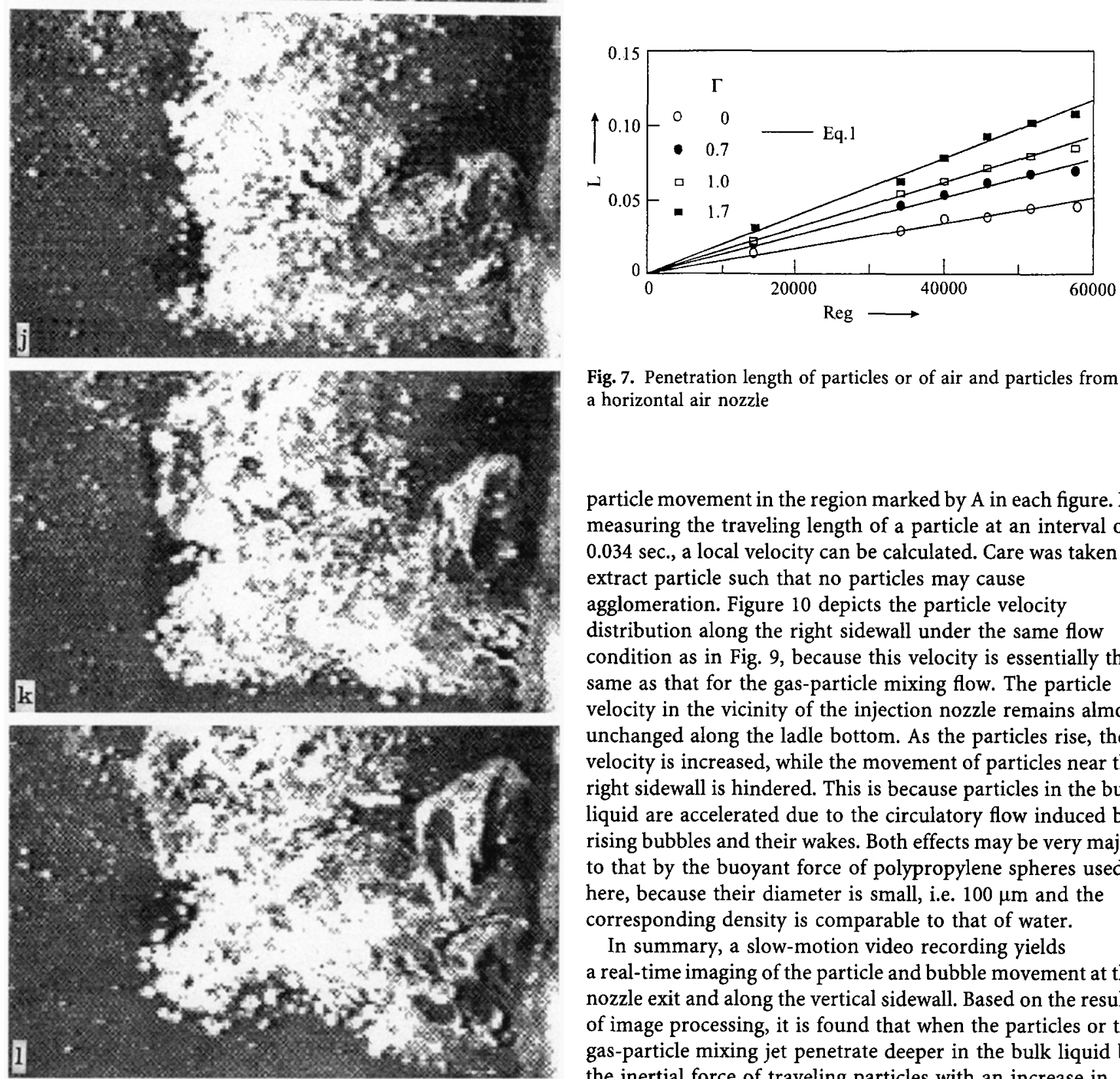

Fig. 7. Penetration length of particles or of air and particles from a horizontal air nozzle

particle movement in the region marked by $A$ in each figure. By measuring the traveling length of a particle at an interval of $0.034 \mathrm{sec}$., a local velocity can be calculated. Care was taken to extract particle such that no particles may cause agglomeration. Figure 10 depicts the particle velocity distribution along the right sidewall under the same flow condition as in Fig. 9, because this velocity is essentially the same as that for the gas-particle mixing flow. The particle velocity in the vicinity of the injection nozzle remains almost unchanged along the ladle bottom. As the particles rise, their velocity is increased, while the movement of particles near the right sidewall is hindered. This is because particles in the bulk liquid are accelerated due to the circulatory flow induced by rising bubbles and their wakes. Both effects may be very major to that by the buoyant force of polypropylene spheres used here, because their diameter is small, i.e. $100 \mu \mathrm{m}$ and the corresponding density is comparable to that of water.

In summary, a slow-motion video recording yields a real-time imaging of the particle and bubble movement at the nozzle exit and along the vertical sidewall. Based on the results of image processing, it is found that when the particles or the gas-particle mixing jet penetrate deeper in the bulk liquid by the inertial force of traveling particles with an increase in the particle flow rate, a homogeneous particle distribution is exhibited, resulting in more effective mixing. Therefore, the penetration depth of particles or gas-particles plays an important role in the mixing performance of three phases (gas, liquid and particles). 

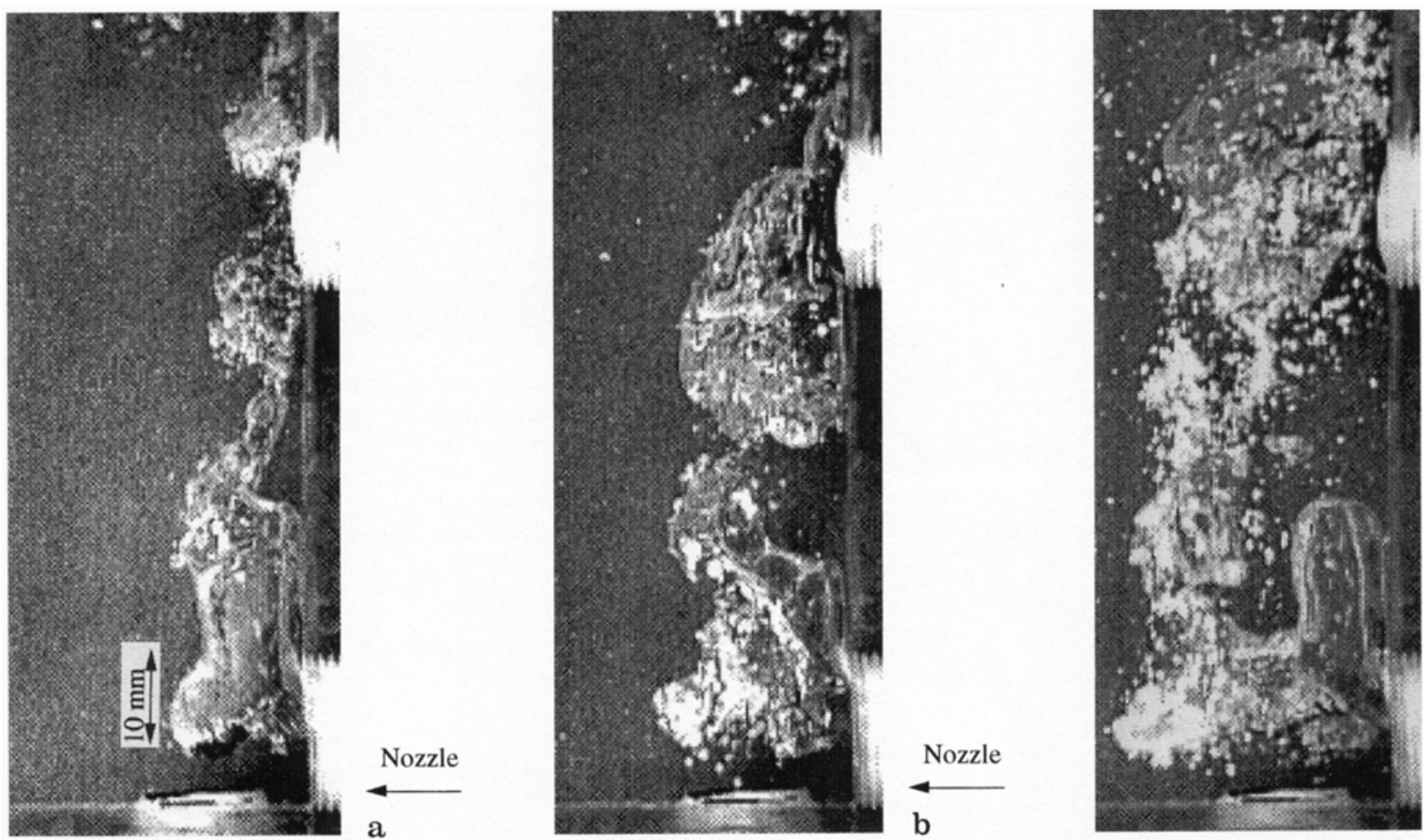

Nozzle a

Fig. 8a-c. Image processing of particle distribution in an air bubble for $Q_{g}=3.51 \times 10^{-4} \mathrm{~m}^{3} / \mathrm{s}$ from a horizontal air nozzle at the lower end of right sidewall, $H_{n} / H=0.05$
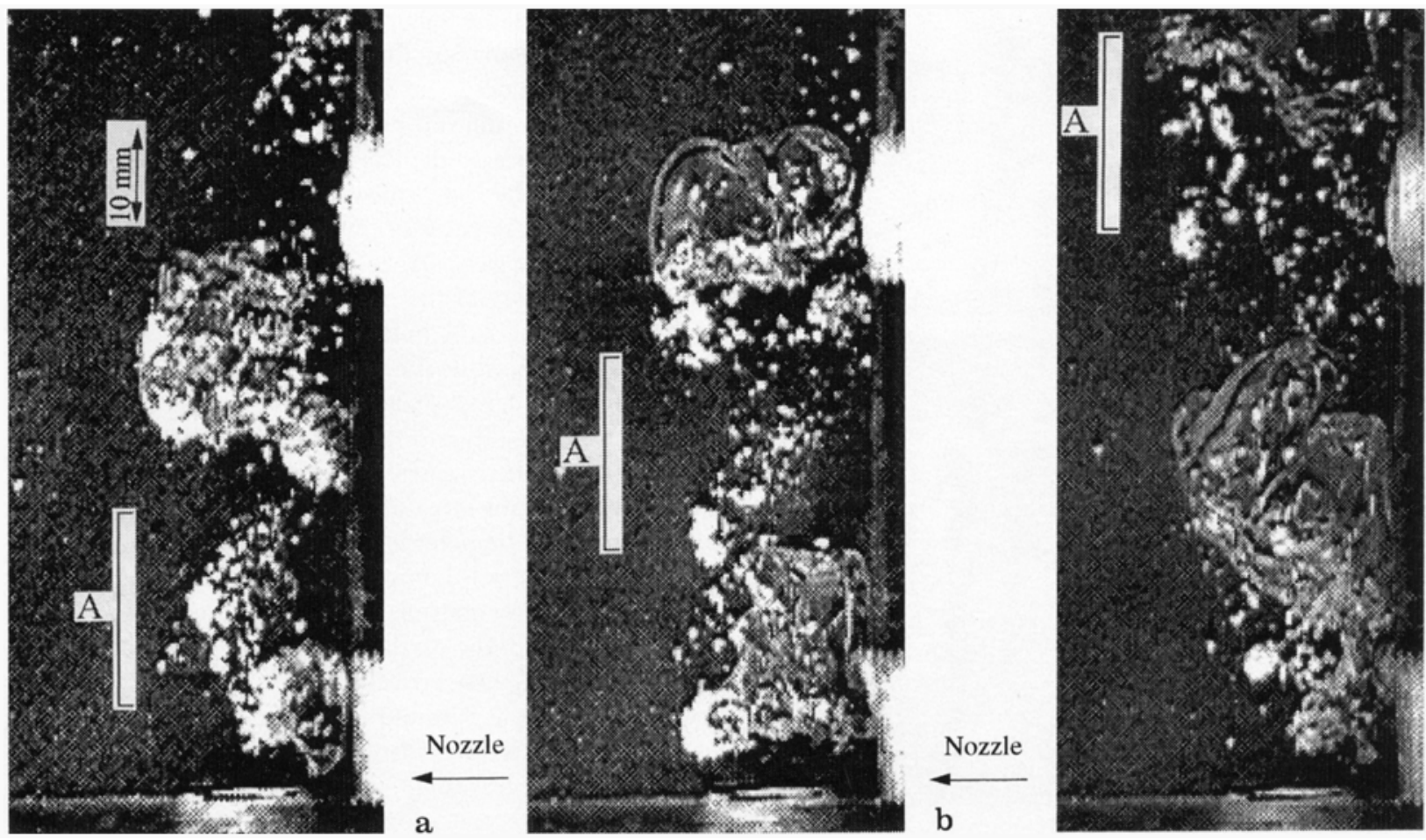

Nozzle

b

c

Fig. 9a-c. Image processing of bubble and particle behavior along sidewall for $Q_{g}=3.51 \times 10^{-4} \mathrm{~m}^{3} / \mathrm{s}$ and $Q_{S}=10.00 \times 10^{-6} \mathrm{~m}^{3} / \mathrm{s}$ from a horizontal air nozzle at the lower end of right sidewall, $H_{n} / H=0.05$ 


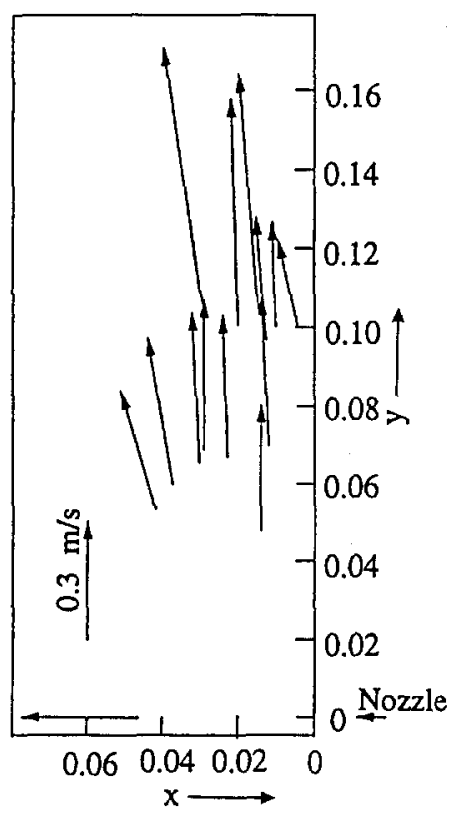

Fig. 10. Velocity profiles of gas-particle-liquid mixing flow along right sidewall for $Q_{g}=3.51 \times 10^{-4} \mathrm{~m}^{3} / \mathrm{s}$ and $Q_{S}=10.00 \times 10^{-6} \mathrm{~m}^{3} / \mathrm{s}$

\section{5}

\section{Summary}

Image processing and a direct photographic method have been employed to investigate the bubble morphology and mechanics of mixing of air-liquid-particle phases during the air-particle injection into a slender tank. The results obtained are summarized as follows:

(1) The behavior of particles and gas being injected into the liquid through a horizontal nozzle varies with their flow rates, $Q_{g}$ and $Q_{S}:$ (a) When both $Q_{g}$ and $Q_{S}$ are low, particles stick on the inner surface of the bubble, (b) When only $Q_{S}$ is high, particles break through the bubble surface, and (c) When only $Q_{g}$ is high or both $Q_{g}$ and $Q_{S}$ are high, a solid-gas, two-phase jet is formed. This jet varies in shape with time. The penetration length of the particles or jet is increased with an increase in the flow rates of gas and particles. Its magnitude can be predicted or correlated by Eq. (1).

(2) A real-time image processing with the aid of a slow-motion video recording discloses the bubble and particle behavior along the ladle sidewalls, and the particle velocity distribution can be quantitatively determined. A better particle-liquid mixing is achieved when the particles or the solid-gas, two-phase jet penetrate deeper into the bulk liquid, resulting in a homogeneous mixing.

\section{References}

Engh TA (1979) Penetration of particle-gas jets into liquids. Ironmaking and Steelmaking 6: 268-273

Grevet J; Szekely J; El-Kaddah N (1982) An experimental and theoretical study of gas-bubble driven circulation systems. Int J Heat Mass Transfer 25: 487-497

Mazumdar D; Guthrie RIL (1985) Hydrodynamic modeling of some gas injection procedures in ladle metallurgy operations. Metallurgical Transactions B. 16B: 83-90

Sahai Y; Guthrie RIL (1982a) Hydrodynamics of gas stirred metals. Part I: gas/liquid coupling. Metallurgical Transactions B 13B: 193-202

Sahai Y; Guthrie RIL (1982b) Hydrodynamics of gas stirred metals. Part I: axisymmetric flows. Metallurgical Transactions B 13B: 203-211

Sahai Y; Guthrie RIL (1986) Recent advances in the hydrodynamics of metallurgical processing. In: Advances in Transport Processes. (eds. Mazumdar AS; Mashelakar RA). pp 1-48, New York: A Halsted Press Book

Szekely J; Wang HJ; Kiser KM (1976) Flow pattern velocity model of an argon-stirred ladle. Metallurgical Transactions B. 7B: 287-295

Torii S; Yang WJ (1992) Melt-particle mixing in gas-stirred ladles with throughflow, Exp Fluids 13: 37-42

Torii S; Yang WJ (1991) Bubble and particle behavior in air-solid injection. In: Transport Phenomena Science and Technology (ed. Bu-Xuan Wang). pp 341-346, $\square$ Higher Education Press

Torii S; Wang WJ (1993) Image processing in gas-liquid-particle mixing flow. In: Image in Transport Processes (ed. Sideman $S$ and Hijikata K). pp 351-363, $\square$ Begell House, Inc

Torii S; Yang WJ (1995) Penetration of an injected gas-particle mixture into a melt (to appear in the Proceedings of the Symposium of Flow Visualization and Image Processing of Multiphase Systems, ASME, Aug. 1995) 